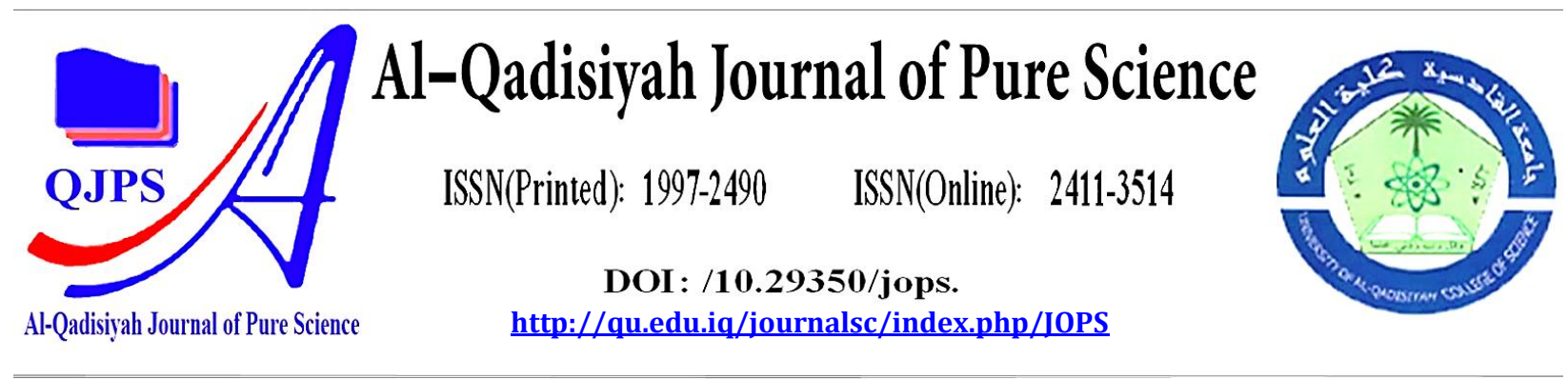

\title{
Strongly N-extending modules
}

\section{Authors Names \\ a. Saad Abdulkadhim Al-Saad \\ b. Darya Jabar Abdul-Kareem}

\section{Article History}

Received on: $12 / 6 / 2021$

Revised on: $30 / 7 / 2021$

Accepted on: 8/8/2021

DOI: https://doi.org/10.29350/ jops.2021.26. 4.1350

\section{ABSTRACT}

Relative extending modules and relative (quasi-)continuous modules were introduced and studied by Oshiro as a generalizations of extending modules and (quasi-) continuous respectively. On other hand, Oshiro, Rizvi and Permouth introduced $\mathrm{N}$-extending and $\mathrm{N}$-(quasi-) continuous modules depending $\quad \mathcal{A}=\mathcal{A}(\mathrm{N}, \mathrm{M})=\{\mathrm{A} \subseteq \mathrm{M} \mid \exists \mathrm{X} \subseteq \mathrm{N}, \exists$ fHom $(\mathrm{X}, \mathrm{M})$ such that $\mathrm{f}(\mathrm{X})$ is essential in $\mathrm{A}\}$ where $\mathrm{N}$ and $\mathrm{M}$ are modules. $\mathcal{A}(\mathrm{N}, \mathrm{M})$ is closed under submodules, essential extension and isomorphic image. A module $\mathrm{M}$ is $\mathrm{N}$ extending if for each submodule $\mathrm{A} \in \mathcal{A}(\mathrm{N}, \mathrm{M})$, there is a direct summand $\mathrm{B}$ of $\mathrm{M}$ such that $\mathrm{A}$ is essential in $\mathrm{B}$. Moreover, a module $\mathrm{M}$ is strongly extending if every submodule is essential in a stable (equivalently, fully invariant) direct summand of $\mathrm{M}$.

In this paper, we introduce and study classes of modules which are proper stronger than that of $\mathrm{N}$-extending modules and $\mathrm{N}$-(quasi-)continuous modules. Many characterizations and properties of these classes are given.

\section{Introduction}

Let $R$ be a ring with identity and $M$ be a left unitary $R$-module. A nonzero submodule $N$ of $M$ is essential if every non-zero submodule of $M$ has non-zero intersection with $N$. Also, a submodule $N$ of $M$ is closed in $M$, if it has no proper essential extensions in $M$. Also, Let $N$ be a submodule of $M$, a relative complement of $N$ in $M$ is any submodule $N^{\prime}$ of $M$ which is maximal with respect to the property that $N \cap N^{\prime}=0$. For details of these concepts see [3]. Recall that a module $M$ is extending if, every submodule of $M$ is essential in a direct summand of $M$.

The notion of the extending modules and their generalizations studied extensively by many authors. Oshiro in [7], introduced relative extending modules a generalization of the concept of extending modules. Following [7], let $\mathcal{A}$ be subfamily of the family of all submodules of an R-module $M$, a module $M$ is $\mathcal{A}$-extending if every submodule which with belong to $\mathcal{A}$ is essential in a direct summand of $M$. On the other hand, K. Oshiro, S. Riziv and S.Permouth in [6], 
introduced $N$-extending modules, depending on the family $\mathcal{A}(N, M)=\{A \subseteq M \mid \exists X \subseteq N, \exists f \in$ $\operatorname{Hom}(X, M), f(X)$ is essential in $A\}$ where $N, M$ are modules. They called a module $M$ is $N$ extending (or $M \operatorname{has} \mathcal{A}(N, M) \mathrm{C}_{1}$ ) if for each $A \in \mathcal{A}(N, M)$, there exists $A^{\prime}$ which is a direct summand of $M$ such that $A$ is essential in $A^{\prime}$.

The author in [2] introduced and studied a class of modules which is stronger than extending modules. A module $M$ is strongly extending if, every submodule in $M$ is essential in a stable (equivalently, fully invariant) direct summand of $M$.

In paper, we introduce and study relative strongly extending modules which are stronger concepts of relative extending modules.

\section{Strongly $N$-extending modules.}

Recall that a submodule $N$ of a module $M$ is fully invariant if $f(N) \subseteq N$ for each $f \in \operatorname{End}_{R}(M)$ and a module $M$ is called Duo if every submodule of $M$ is fully invariant [8]. A submodule $N$ of a module $M$ is called stable if, $f(N)) \subseteq N$ for each homomorphism $f: N \rightarrow M$. A module $M$ is called fully stable if each submodule of $M$ is stable [1].

Definition (1.1): Let $N$ be a module. A module $M$ is said to be strongly $N$-extending if for each submodule $A$ of $M$ with $A \in \mathcal{A}(N, M)$, is essential in a stable direct summand of $M$.

\section{Remarks and Examples (1.2):}

(1) Every strongly extending is strongly $N$-extending for each module $N$. But the converse is not true. For example, the $Z$-module $M=Z \oplus Z$ is $Z_{3}$-extending which is not strongly extending.

(2) Every strongly $N$-extending is $N$-extending. But the converse is not true in general. For example, the $Z$-module $M=Z_{2} \oplus Z$ is $Z_{2}$ - extending [6], which is not strongly $Z_{2}$-extending. Since $Z_{2} \oplus 0$ is closed submodule of $M$ and belong to $\mathcal{A}\left(Z_{2}, M\right)$. Let $f: Z_{2} \oplus(0) \rightarrow Z_{2} \oplus Z$ by $f(x, 0)=(0, x)$ for each $[\bar{x}, 0) \in Z_{2} \oplus(0)$. $f$ is $Z$-homomorphism while $f\left(Z_{2} \oplus(0)\right) \nsubseteq Z_{2} \oplus(0)$ so $Z_{2} \oplus 0$ is not stable submodule of $M$.

(3) Every uniform module is strongly $N$-extending for each R-module $N$. In particular, $Z$ as $Z$ module is strongly $Z_{2}$-extending.

(4) But the converse of (3) every uniform module is strongly $\mathrm{N}$-extending is not true. For example, $Z_{6}$ as $Z$-module is strongly $Z_{2}$-extending which is not uniform.

(5) Since the set of all submodules of a modules $M$ is coincide with $\mathcal{A}(M, M)$ [6]. The following statements are equivalent:

(i) $M$ is strongly extending;

(ii) $M$ is strongly $M$ - extending;

(iii) $M$ is strongly $N$-extending for every module $N$.

The proof is direct from the fact " the set of all submodules of a modules $\mathrm{M}$ is coincide with $\mathcal{A}(\mathrm{M}, \mathrm{M})[6] "$. 
It is proved in [2] that a module $M$ is stronglyextending if and only if every closed submodule of $M$ is a stable direct summand of $M$.This result leads us to give the following characterization of strongly $N$-extending modules.

Proposition (1.3): A module $M$ is strongly $N$-extending if and only if every closed submodule of $M$ which with belongs to $\mathcal{A}(N, M)$ is a stable direct summand.

Proof: $(\Rightarrow)$ Suppose that $M$ is strongly $N$-extending module. Let $K$ be a closed submodule of $M$ with $K \in \mathcal{A}(N, M)$. Since $M$ is strongly $N$-extending, then there exists a stable direct summand $B$ of $M$ such that $K$ is essential in $B$. But $K$ is closed submodule of $M$, hence $K=B$ (i.e.) $K$ is a stable direct summand of $M$.

$(\Longleftarrow)$ Let $A$ be a submodule of $M$ with $A \in \mathcal{A}(N, M)$. Thus, by Zorn's lemma, there exists a closed submodule $H$ of $M$ such that $A$ is essential in $H$. Since $\mathcal{A}(N, M)$ is closed under essential extension, so $H \in \mathcal{A}(N, M)$. By hypothesis, $H$ is a stable direct summand of $M$. Therefore, $M$ is strongly $N$-extending. $\square$

The next results give us characterizations of strongly $N$-extending modules.

Proposition (1.4): A module $M$ is strongly $N$-extending if and only if for each submodule $A$ of $M$ with $A \in \mathcal{A}(N, M)$, there is a direct decomposition $M=M_{1} \oplus M_{2}$ such that $\mathrm{A} \subseteq M_{1}$ where $M_{1}$ is a stable submodule of $M$ and $A \oplus M_{2}$ is essential of $M$.

Proof: $(\Longrightarrow)$ Suppose that $M$ is strongly $N$-extending $R$-module. Let $A$ be a submodule of $M$ with $A \in \mathcal{A}(N, M)$.Thus $A$ is essential in a stable direct summand (say) $K$ of $M$ (i.e.) $M=K \oplus K_{1}$, where $K_{1}$ is a submodule of $M$. Also, since $A$ is essential in $K$ and $K_{1}$ is essential in $K_{1}$, thus $A \oplus K_{1}$ is essential in $K \oplus K_{1}=M$. Hence $A \oplus K_{1}$ is essential submodule of $M$.

$(\Longleftarrow)$ Let $A$ be a submodule of $M$ with $A \in \mathcal{A}(N, M)$. By hypothesis, there is a direct decomposition $M=M_{1} \oplus M_{2}$ such that $A \subseteq M_{1}$ where $M_{1}$ is a stable submodule of $M$ and $A \oplus M_{2}$ is essential in $M$. We claim that $A$ is essential in $M_{1}$. Let $K$ be a nonzero submodule of $M_{1}$, hence $K$ is a submodule of , so $\left(A \oplus M_{2}\right) \cap K \neq(0)$ (since $A \oplus M_{2}$ is essential in $M$ ). Let $k=a+m_{2}(\neq 0)$, where $k \in K, a \in A$ and $m_{2} \in M_{2}$, thus $m_{2}=k-a$ which implies $m_{2} \in M_{1} \cap M_{2}=(0)$, therefore $0 \neq k=a \in K \cap A$, then $K \cap A \neq(0)$, hence $A$ is essential in $M_{1}$. Thus $M$ is strongly $N$-extending. $\square$

Following [2], every fully invariant direct summand is stable. By using this fact, we have directly the following characterization of strongly $N$-extending modules.

Proposition (1.5): A module $M$ is strongly $N$-extending if and only if every submodule of $M$ with belong $\mathcal{A}(N, M)$ is essential in a fully invariant direct summand of $M . \square$

Remark (1.6): From above Proposition, it can be restated that all results with stable direct summand being replaced by fully invariant direct summand.

In the following results, we discuss when a submodule of strongly $N$-extending module is strongly $N$-extending.

Proposition (1.7): A closed submodule of strongly $N$-extending is strongly $N$-extending. 
Proof: Let $H$ be a closed submodule of strongly $N$-extending module $M$. Let $K$ be a closed submodule of $H$ with $K \in \mathcal{A}(N, H)$. Since $H$ is closed submodule of $M$, then $K$ is closed of $M$. Also, $K \in \mathcal{A}(N, M)$. Now, since $M$ is strongly $N$-extending, thus $K$ is a stable direct summand of $M$. But $K \subseteq H$, then $K$ is direct summand of $H$. Also, we claim that $K$ is a stable submodule of $H$. Let $f: K \rightarrow H$ be any homomorphism and consider the sequence $\stackrel{f}{\rightarrow} H \stackrel{i}{\rightarrow} M$, where $i$ is the inclusion mapping. Then $(i \circ f): K \rightarrow M$, and since $K$ is stable of $M$, then $(i \circ f)(K) \subseteq K$. So $f(K) \subseteq K$.Then $K$ is a stable direct summand of $H$. Therefore $H$ is strongly $N$-extending. $\square$

Corollary (1.8): A direct summand of strongly $N$-extending module is strongly $N$-extending. $\square$

Corollary (1.9): A direct summand $A$ of strongly $N$-extending module with $A \in \mathcal{A}(N, M)$ is strongly extending. $\square$

Proposition (1.10): Every submodule $H$ of strongly $N$-extending module $M$ and with the property that the intersection of $H$ with any stable direct summand of $M$ is stable direct summand of $H$, is strongly $N$-extending.

Proof: Let $A$ be a submodule of $H$ with $A \in \mathcal{A}(N, H)$. Since $M$ is strongly $N$-extending and $A$ is a submodule of $M$, then there is a stable direct summand $K$ of $M$ such that $A$ is essential in $K$ of $M$. But $A \subseteq K \cap H \subseteq K$, thus $A$ is essential in $K \cap H$ and by hypothesis, $K \cap H$ stable direct summand of $H$. Hence $H$ is strongly $N$ - extending. $\square$

It is well known that a direct sum of (strongly) extending modules need not be (strongly) extending ([2])[9].

Here, we see that, a direct sum of strongly $N$-extending need not be strongly $N$-extending. In fact, $Z$ and $Z_{2}$ are $Z_{2}$-extending as $Z$-modules but we can concluded that From remarks and examples (1.2) (2), $Z_{2} \oplus Z$ is not strongly $Z_{2}$-extending as $Z$-module.

In the next result, we obtain when a direct sum of strongly $\mathrm{N}$ - extending module is strongly $\mathrm{N}$ extending module.

Theorem(1.11):Let $M=M_{1} \oplus M_{2}$ where $M_{1}$ and $M_{2}$ are strongly $N$-extending. Then $M$ is strongly $N$-extending if and only if every closed submodule $K$ belong to $\mathcal{A}(N, M)$ with $K \cap M_{1}=0$ or $K \cap M_{2}=0$ is stable direct summand.

Proof: The necessity condition is valid by Proposition (1.8). Conversely, let $L$ be a closed submodule of $M$ with $L \in \mathcal{A}(N, M)$. By Zorn's lemma, there exists a closed submodule $H$ in $L$ such that $L \cap M_{2}$ is essential in $H$. Since $L$ is a closed submodule of $M$, so $H$ is a closed submodule of $M$. Clearly since $L \cap M_{2}$ is essential in $H$ and $M_{1}$ is essential in $M_{1}$ so $\left(L \cap M_{2}\right) \cap$ $M_{1}$ is essential in $H \cap M_{1}$ Since $\left(L \cap M_{2}\right) \cap M_{1}$ is essential in $H \cap M_{1}$ and $(L \cap M 2) \cap M_{1}=0$ so $H \cap M_{1}=0$. Then by hypothesis, $H$ is a stable direct summand of $M$. Let $M=H \oplus H^{\prime}$ for some submodule $H^{\prime}$ of $M$. But $L \in \mathcal{A}(N, M)$ and $H$ is a submodule of $L$, then $H \in \mathcal{A}(N, M)$ (since $\mathcal{A}(N, M)$ is closed under submodules). Now, $L=L \cap M=L \cap\left(H \oplus H^{\prime}\right)=H \oplus\left(L \cap H^{\prime}\right)$. So $\left(L \cap H^{\prime}\right)$ is closed in $M$ with $L \cap H^{\prime} \in \mathcal{A}(N, M)$ (Since $\left(L \cap H^{\prime}\right)$ is a submodule of $L$ and $L \in \mathcal{A}(N, M)$. Also, $\left(L \cap H^{\prime}\right) \cap M_{2}=0$. By hypothesis, $\left(L \cap H^{\prime}\right)$ is a stable direct summand of $M$, and hence of $H^{\prime}$ (since $\left(L \cap H^{\prime}\right) \subseteq H^{\prime}$ ). Thus, $H^{\prime}=\left(L \cap H^{\prime}\right) \oplus K$, where $K$ is a submodule of $H^{\prime}$. Now, $\left.M=H \oplus H^{\prime}=H \oplus\left(L \cap H^{\prime}\right) \oplus K\right)=\left(H \oplus\left(L \cap H^{\prime}\right) \oplus K\right)=L \oplus K$. It follows that $L$ is a 
direct summand of $M$. Since $H$ and $L \cap H^{\prime}$ are stable submodule of $M$ and $L=H \oplus\left(L \cap H^{\prime}\right)$, then $L$ is stable of $M$ [1]. So $L$ is a stable direct summand of $M$. Theretofore, $M$ is strongly $N$ extending. $\square$

Following [6], if $M$ be an R-module and $0 \rightarrow \mathrm{N}^{\prime} \rightarrow \mathrm{N} \rightarrow \mathrm{N}^{\prime \prime} \rightarrow 0$ be an exact sequence of modules, then $\mathcal{A}\left(N^{\prime}, M\right) \cup \mathcal{A}\left(N^{\prime \prime}, M\right) \subseteq \mathcal{A}(N, M)$.

Proposition (1.12): Let $0 \rightarrow N^{\prime} \rightarrow N \rightarrow N^{\prime \prime} \rightarrow 0$ be an exact sequence. Then, a module $M$ is strongly $N$-extending if and only if $M$ is strongly $N^{\prime}$-extending and $M$ is strongly $N^{\prime \prime}$-extending.

Proof: $(\Longrightarrow)$ It is clear by using the fact $\mathcal{A}\left(N^{\prime}, M\right) \cup \mathcal{A}\left(N^{\prime \prime}, M\right) \subseteq \mathcal{A}(N, M)$.

$(\Leftarrow)$ Suppose that $M$ is strongly $N^{\prime}$-extending and strongly $N^{\prime \prime}$-extending. Since $0 \rightarrow N^{\prime} \rightarrow N \rightarrow$ $N^{\prime \prime} \rightarrow 0$ is exact sequence. So one can assume that $N^{\prime} \subseteq N$ and $N^{\prime \prime}=N / N^{\prime}$. Let $K \in \mathcal{A}(N, M)$, then there exists $L$ be a submodule of $N$ and there exists $g \in \operatorname{Hom}(L, M)$ such that $g(L)$ is essential in $K$. Let $K^{\prime}$ be a submodule of $K$ which is closure of the submodule $g\left(L \cap N^{\prime}\right)$. Since $M$ is strongly $N^{\prime}$-extending, then $K^{\prime}$ is a stable direct summand of $M$. Let $M=K^{\prime} \oplus K^{\prime \prime}$, for some submodule $K^{\prime \prime}$ of $M$ and $K=K^{\prime} \oplus\left(K \cap K^{\prime \prime}\right)$. Let $\pi: K \rightarrow K \cap K^{\prime \prime}$ denote the canonical projection. Define $\theta:\left(L+N^{\prime}\right) / N^{\prime} \rightarrow \operatorname{Mby} \theta\left(x+N^{\prime}\right)=\pi g(x)$, for all $x \in L$. Note that given $x \in L$, if $x \in N^{\prime}$ then $g(x) \in g\left(L \cap N^{\prime}\right) \subseteq K^{\prime}$ and hence $\theta\left(x+N^{\prime}\right)=\pi g(x)=0$. Thus, $\theta$ is well-defined and clearly $\theta \in \operatorname{Hom}\left(\left(L+N^{\prime}\right) / N^{\prime}, M\right)$. Now let $0 \neq y \in K \cap K^{\prime \prime}$. Then $0 \neq y r=g(u)$ for some $r \in R, u \in L$.Since $g(u) \in K \cap K^{\prime \prime}$ it follows that $0 \neq y r=$ $g(u)=\pi g(u)=\theta\left(u+N^{\prime}\right)$. Thus $\theta\left(\left(L+N^{\prime}\right) / N^{\prime}\right)$ is essential in the closed submodule $K \cap K^{\prime \prime}$ of the strongly $N^{\prime \prime}$-extending module $M$. Hence $K \cap K^{\prime \prime}$ is a stable direct summand of $M$. Since $K^{\prime}$ and $K \cap K^{\prime \prime}$ are direct summand of $M$ and $K=K^{\prime} \oplus\left(K \cap K^{\prime \prime}\right)$, then $K$ is direct summand of $M$. In other, direction, since $K^{\prime}$ and $K \cap K^{\prime \prime}$ are stable of $M$ and $K=K^{\prime} \oplus(K \cap$ $\left.K^{\prime \prime}\right)$, then $K$ is a stable of $M$.So $K$ is stable direct summand of $M$. Therefore, $M$ is strongly $N$ extending. $\square$

Relative (quasi-)continuous modules have been considered by several authors. In [4], S. Dogrouz considers (quasi-)continuous modules relative to a class of modules. On other hand, Lopez, Oshiro and Rizivi in [6] introduced (quasi-)continuous modules relative a given Rmodule $N$. A module $M$ is called $N$-continuous if satisfies $N$-extending and $\mathcal{A}(N, M) \mathrm{C}_{2}$ : Every submodule of $M$ which is belong in $\mathcal{A}(N, M)$, which is isomorphic to a direct summand of $M$ is a direct summand of $M$. A module $M$ is called $N$-quasi-continuous if satisfies $N$-extending and $\mathcal{A}(N, M) \mathrm{C}_{3}$ : If two direct summand $A$ and $X$ of $M$ which $A$ belong in $\mathcal{A}(N, M)$ have zero intersection, then their sum is a direct summand of $M$.

Here, we introduce relative strongly (quasi-)continuous modules.

Firstly, we can consider the following conditions for modules $M$ and $N$ :

$\mathcal{A}(N, M) \mathrm{SC}_{1}$ : Every submodule of $M$ which is belong in $\mathcal{A}(N, M)$ is essential in a stable direct summand of $M$.

$\mathcal{A}(N, M) \mathrm{SC}_{2}$ : Every submodule of $M$ which is belong in $\mathcal{A}(N, M)$, which is isomorphic to a direct summand of $M$ is a stable direct summand of $M$. 
$\mathcal{A}(N, M) \mathrm{SC}_{3}$ : If two direct summand $A$ and $X$ of $M$ which $A$ belong in $\mathcal{A}(N, M)$ have zero intersection, then their sum is a stable direct summand of $M$.

Definition (1.13): A module $M$ is said to be strongly $N$-continuous if $M$ satisfies, $\mathcal{A}(N, M) \mathrm{SC}_{1}$ and $\mathcal{A}(N, M) \mathrm{SC}_{2}$.

Definition (1.14): A module $M$ is said to be strongly $N$-qausi- continuous if $M$ is satisfies, $\mathcal{A}(N, M) \mathrm{SC}_{1}$ and $\mathcal{A}(N, M) \mathrm{SC}_{3}$.

\section{Examples (1.15):}

(1) Every strongly $N$-(quasi-)contenuous is $N$-(quasi-)continuous and the converse is not true in general. For example, $M=Z_{2} \oplus Z$ is $Z_{2}$-(quasi)-continuous as $Z$-module [6] which is not strongly $Z_{2}$-quasi-continuous $Z$-module.

(2) Every strongly $N$-continuous module is strongly $N$-quasi-continuous and the converse is not true in general. For example, $Z$ as $Z$-module is strongly $Z$-quasi-continuous but it is not strongly $Z$-continuous, since $Z$ as $Z$-module does not satisfy $\mathcal{A}(N, M) \mathrm{SC}_{2}$ condition. In fact, $3 Z \in \mathcal{A}(Z, Z)$ and $3 Z \cong Z$ and is direct summand of $Z$. But $3 Z$ is not stable direct summand of $Z$.

Recall that a module $\mathrm{M}$ is SS-module if every direct summand of $\mathrm{M}$ is stable [2] which is equivalent to weakly Duo module [8], that is, every direct summand is fully invariant. We introduce the following useful concept.

Definition (1.16): Let $M$ and $N$ are modules. $M$ is called SS- $N$-module if, every direct summand $D$ of $M$ with $D \in \mathcal{A}(N, M)$ is a stable.

It is clear that strongly $N$-extending modules, uniform modules, SS-modules, Duo modules and fully stable modules are examples of SS- $N$-module.

\section{Remarks and Examples (1.17):}

(1) Every SS-module is SS- $N$-extending for each module $N$. But the converse is not true in general. For example, the Z-module $M=Z \oplus Z$ is SS- $N$-module for each semisimple module $N$. But $M$ is not SS-module, in fact, $Z \oplus(0)$ is direct summand of $M$ which is not stable submodule of $M=Z \oplus Z$.

(2) A direct sum of SS- $N$-module need not be SS- $N$-module. For example, consider $Z$ and $Z_{2}$ as $Z$ modules. Since $Z$ and $Z_{2}$ are uniform $Z$-modules, then they are SS- $Z_{2}$-module. But $M=Z_{2} \oplus Z$ as $Z$-module is not SS-Z $Z_{2}$-module. In fact the only proper direct summand of $M$ are $(0) \oplus$ $(0), Z_{2} \oplus(0),(0) \oplus Z$ and $Z \oplus Z_{2}$. One can easily check that $\mathcal{A}\left(Z_{2}, M\right)=\left\{(0), Z_{2} \oplus(0)\right\}$. But $Z_{2} \oplus(0)$ is not stable submodule of $M$.

(3)A direct summand of SS- $N$-module is SS- $N$-module.

Proof: Let $M$ be SS- $N$-module and $H$ be a direct summand of $M$. Let $K$ be a direct summand of $H$ with $K \in \mathcal{A}(N, H)$. Since $H$ is a direct summand of $M$, then $K$ is a direct summand of $M$. Also $K \in \mathcal{A}(N, M)$. Now since $M$ is SS- $N$-module, thus $K$ is a stable submodule of $M$. But, $K \subseteq H$, then $K$ is direct summand of $H$. We claim that $K$ is stable of $H$. Let $f: K \rightarrow H$ be any 
homomorphism. Thus $(i \circ f): K \rightarrow M$ where $i: H \rightarrow M$ is inclusion mapping and so ( $i \circ$ $f)(K) \subseteq K$. (i.e. ) $f(K) \subseteq K$. Thus $K$ is stable submodule of $H$. Hence $H$ is SS- $N$-module. $\square$

In the following lemmas, we give a characterization of modules satisfy the conditions $\mathcal{A}(N, M) \mathrm{SC}_{1}, \mathcal{A}(N, M) \mathrm{SC}_{2}$ and $\mathcal{A}(N, M) \mathrm{SC}_{3}$.

Lemma (1.18): A module $M$ has $\mathcal{A}(N, M) \mathrm{SC}_{1}$ condition if and only if $M$ has $\mathcal{A}(N, M) \mathrm{C}_{1}$ condition and $M$ has SS- $N$-extending.

Proof: $(\Longrightarrow)$ Let $M$ has $\mathcal{A}(N, M) \mathrm{SC}_{1}$ condition.Then, clearly $M$ satisfy $\mathcal{A}(N, M) \mathrm{C}_{1}$ condition. Let $D$ be a direct summand of $M$ such that $D \in \mathcal{A}(N, M)$. So, $D$ is closed which belong to $\mathcal{A}(N, M)$, thus by Proposition (1.3), $D$ is stable.

$(\Longleftarrow)$ Let $H$ be a submodule of $M$ with $H \in \mathcal{A}(N, M)$. Then by $\mathcal{A}(N, M) \mathrm{C}_{1}$ property of $M, H$ is essential in a direct summand $D$ of $M$. But $D \in \mathcal{A}(N, M)$, since $\mathcal{A}(N, M)$ is closed under essential extension. So by SS- $N$-module $D$ is a stable. Therefore, $M$ has $\mathcal{A}(N, M) \mathrm{SC}_{1}$.

Lemma (1.19): A module $M$ has $\mathcal{A}(N, M) \mathrm{SC}_{2}$ condition if and only if $M$ has $\mathcal{A}(N, M) \mathrm{C} 2$ condition and $M$ is SS- $N$-module.

Proof: $(\Longrightarrow)$ Let $M$ has $\mathcal{A}(N, M) \mathrm{SC}_{2}$ condition. Then, clearly, $M$ has $\mathcal{A}(N, M) \mathrm{C}_{2}$ condition. Let $H$ is a direct summand of $M$ with $H \in \mathcal{A}(N, M)$. Since $H \cong H$ and hence by $\mathcal{A}(N, M) \mathrm{SC}_{2}$ property, $H$ is a stable submodule of $M$. So $M$ is SS- $N$-module.

$(\Leftarrow)$ Let $H$ be a submodule of $M$ with $H \in \mathcal{A}(N, M)$ such that $H \cong D$ where $D$ is a direct summand of $M$. Thus, by $\mathcal{A}(N, M) \mathrm{C}_{2}$ property $H$ is a direct summand of $M$. Also, by SS- $N$-module property of $M$ we have $H$ is a stable of $M$. Therefore, $M$ has $\mathcal{A}(N, M) \mathrm{SC}_{2}$ condition. $\square$

Lemma (1.20): A module $M$ has $\mathcal{A}(N, M) \mathrm{SC}_{3}$ condition if and only if $M$ has $\mathcal{A}(N, M) \mathrm{C}_{3}$ condition and $M$ is SS- $N$-module.

Proof: $\Longrightarrow$ ) Clearly every module $M$ satisfies $\mathcal{A}(N, M) \mathrm{SC}_{3}$ has $\mathcal{A}(N, M) \mathrm{C}_{3}$. Let $H$ be a direct summand of $M$ with $H \in \mathcal{A}(N, M)$. Let $D=(0)$. So $D$ and $H$ are direct summands of $M$ such that $H \cap D=(0)$. Thus, by $\mathcal{A}(N, M) \mathrm{SC}_{3}$ property $H \oplus D=(0) \oplus H=H$ is stable submodule of $M$.Then $M$ is SS- $N$-module.

$(\Longleftarrow$ ) Let $H \in \mathcal{A}(N, M)$ such that $H$ and $K$ are direct summands of $M$ and $H \cap K=(0)$.Thus, by $\mathcal{A}(N, M) \mathrm{C}_{3}$ property for $M, H \oplus D$ is a direct summand of $M$. Also, $H \oplus D \in \mathcal{A}(N, M)($ since $H \oplus D$ is submodule of $H$ and $H \in \mathcal{A}(N, M)$ and since $\mathcal{A}(N, M)$ is closed under submodule). Then by SS- $N$-module $H \oplus D$ stable of $M$. Hence $M$ satisfies $\mathcal{A}(N, M) \mathrm{SC}_{3}$ property. $\square$

By using above three lemmas, we have the following characterizations of strongly $N$-(quasi)continuous modules. The proofs of these propositions are direct.

Proposition (1.21): A module $M$ is strongly $N$-continuous if and only if $M$ is $N$-continuous and $M$ is SS- $N$-module. $\square$

Proposition (1.22): A module $M$ is strongly $N$-quasi-continuous if and only if $M$ is $N$-quasicontinuous and $M$ is SS- $N$-module. $\square$ 
Proposition (1.23): A module $M$ is strongly $N$-continuous if and only if $M$ is satisfies the condition $\mathcal{A}(N, M) \mathrm{SC}_{1}$ and $\mathcal{A}(N, M) \mathrm{C}_{2}$.

Proposition (1.24): A module $M$ is strongly $N$-quasi-continuous if and only if $M$ is satisfies the condition $\mathcal{A}(N, M) \mathrm{SC}_{1}$ and $\mathcal{A}(N, M) \mathrm{C}_{3 .}$.

Proposition (1.25): A module $M$ is strongly $N$-continuous if and only $M$ satisfies the condition $\mathcal{A}(N, M) \mathrm{C}_{1}$ and $\mathcal{A}(N, M) \mathrm{SC}_{2}$.

Proposition (1.26): A module $M$ is strongly $N$-quasi-continuous if and only if $M$ satisfies the condition $\mathcal{A}(N, M) \mathrm{C}_{1}$ and $\mathcal{A}(N, M) \mathrm{SC}_{3 . \square}$

Following [6], a direct summand of $\mathrm{N}$-(quasi)- continuous module is $\mathrm{N}$-(quasi)continuous. So, By Remarks and Example (1.17)(3) and Proposition(1.21) and Proposition (1.22), we assert that the strongly $N$-(quasi-) continuous modules is inherited by direct summands.

Proposition (1.27): A direct summand of strongly $N$-continuous module is strongly $N$ continuous.

Proposition (1.28): A direct summand of strongly $N$-quasi-continuous module is strongly $N$ quasi-continuous

By using the same argument of Proposition (1.12) we have the following results:

Proposition (1.29): Let $0 \rightarrow N^{\prime} \rightarrow N \rightarrow N^{\prime \prime} \rightarrow 0$ be an exact sequence of modules. Then a module $M$ is strongly $N$-continuous if and only if $M$ is strongly $N^{\prime}$ - continuous and strongly $N "$ continuous. $\square$

Proposition (1.30): Let $0 \rightarrow N^{\prime} \rightarrow N \rightarrow N^{\prime \prime} \rightarrow 0$ be an exact sequence of modules. Then $M$ is strongly $N$-quasi-continuous if and only if $M$ is strongly $N^{\prime}$-quasi-continuous and strongly $N^{\prime \prime}$ quasi- continuous. $\square$

\section{References}

[1] Abbas, M.S.: On fully stable modules, Ph.D. Thesis, Univ. of Baghdad, 1991.

[2] Al-Saadi, S.A: S-extending modules and related concepts, Ph. D. Thesis, Mustansiriyah Univ. 2007.

[3] Anderson F.W., and Fuller, K.R.: Rings and Categories of modules, Springer-Verlag. New York 1973.

[4] Dogruoz, S.: Extending modules relative to module classes, Ph. D. Thesis, Uinv. of Glasgow, 1997..

[5] Dung, N.V. ; Hunynh, D.V. ; P.F.Smith and Wisbauer, R.: Extending modules, Pitman Research Notes in Mathematics Series, 313(1994). 
[6] Lopez-permonth, S.R.; Oshiro, K. ; Rizivi, S.T. : On the relative (quasi-) continuity of modules, Comm. Algebra 26(1998), 3497-3510.

[7] Oshrio, K. : Continuous modules and quasi-continuous modules, Osaka J.Math, 20(1983), 681694.

[8] Ozcan, A. C. Duo Modules,Glasgow Math. J.Trust 48(2006)533-545.

[9] Simth, P.F. and Tercan, A. : Continuous and quasi-continuous modules, Houston Math. J 18(1992), 339-348. 